\title{
Computed tomography-guided lung biopsy: Association between biopsy needle angle and pneumothorax development
}

\author{
TAKUMI KURIYAMA $^{1}$, KATSUHIRO MASAGO ${ }^{2}$, YUKI OKADA ${ }^{3}$ and NOBUYUKI KATAKAMI ${ }^{4}$
}

\author{
${ }^{1}$ Department of Radiological and Medical Laboratory Sciences, Nagoya University Graduate School of Medicine, \\ Nagoya 461-8673; ${ }^{2}$ Department of Pathology and Molecular Diagnostics, Aichi Cancer Center, Nagoya 464-8681; Divisions of \\ ${ }^{3}$ Radiological Technology and ${ }^{4}$ Integrated Oncology, Institute of Biomedical Research and Innovation, Kobe 650-0047, Japan
}

Received February 3, 2016; Accepted November 10, 2017

DOI: $10.3892 / \mathrm{mco} .2017 .1512$

\begin{abstract}
Computed tomography (CT)-guided lung biopsy is a well-established diagnostic method for pulmonary lesions. However, the use of this technique often results in pneumothorax development. The aim of the present study was to evaluate the association between biopsy needle angle and pneumothorax development associated with computed tomography-guided lung biopsy. We retrospectively analyzed the results of CT-guided lung biopsies for 325 cases to investigate physical risk factors for pneumothorax development. Biopsy needle angle and patient positioning were included in the analysis. Pneumothorax occurred in 160 of 325 procedures (49.2\%). Discontinuation of the procedure as a result of pneumothorax occurred in 18 of 160 procedures (11.2\%). Upper lung lobe pneumothorax occurred in $40.8 \%$ (58/142), middle lobe in $17.6 \%$ (25/142), and lower lobe pneumothorax occurred in $41.5 \%(59 / 142)$ of these procedures. Discontinuation of the procedure occurred in $2.5 \%$ of the upper lobe (4/160), $0.6 \%$ of the middle lobe $(1 / 160)$, and $8.1 \%$ of the lower lobe $(13 / 160)$ biopsies. Mild pneumothorax occurred in 59.4\% (95/160), moderate in $25.0 \%(40 / 160)$, and severe in $7.5 \%(12 / 160)$ of the affected cases, and biopsy was discontinued in $11.2 \%(18 / 160)$ of the affected cases. When the needle angle was $<90^{\circ}, 40.3 \%$ $(131 / 325)$ of the patients experienced no pneumothorax development, $40.0 \%$ (130/325) developed pneumothorax, and $4.3 \%(14 / 325)$ of the procedures were discontinued. The results showed that use of CT-guided lung biopsy can reduce the rate of pneumothorax development that occurs when other procedures are used. The access route is simple and easy to puncture, and proper use of breath holding reduces diaphragmatic movement.
\end{abstract}

Correspondence to: Mr. Takumi Kuriyama, Department of Radiological and Medical Laboratory Sciences, Nagoya University Graduate School of Medicine, 1-1-20 Daikochou, Higashi-ku, Nagoya 461-8673, Japan

E-mail: t.kuriyama@nagoya-u.jp

Key words: computed tomography-guided biopsy, needle angle, discontinue, pneumothorax, lung cancer

\section{Introduction}

Multidetector computed tomography (MDCT) (1-4) and fluorodeoxyglucose-positron emission tomography (FDG-PET) $(5,6)$ are used to detect and diagnose pulmonary lesions. Early detection improves prognosis, but the pulmonary lesion detection rates are approximately $20 \%$ (1-4) and $<3 \%(5,6)$ for these two methods, respectively. CT-guided lung biopsy is a well-established method for pulmonary lesion diagnosis (7-9). The diagnostic accuracy of this method ranges from 64 to $97 \%$ (10-14). The most common complication associated with CT-guided lung biopsy is pneumothorax development, and the rate of this complication varies widely (i.e., 8-69\%) (10-16). Various investigators have analyzed factors thought to affect the diagnostic accuracies and complication rates for CT-guided lung biopsy (10-20). Risk factors for the development of pulmonary lung biopsy-associated pneumothorax include age, smoking history, lesion volume, lesion position, depth from the pleura to the tumor, biopsy needle angle, and needle thickness (10-20). Operator experience is also a risk factor (17). If the patient develops CT-guided lung biopsy-associated pneumothorax, it is difficult to correct the condition using deaeration (21) or drainage (13). It is, therefore, necessary to decrease the rate of biopsy-associated pneumothorax.

The aim of the present study was to evaluate risk factors, including biopsy needle angle, for CT-guided lung biopsy-associated pneumothorax development. We used a retrospective analysis of a large series of patients with pulmonary lesions who underwent this procedure.

\section{Patients and methods}

Patients. We retrospectively analyzed 325 patients who underwent CT-guided lung biopsy between April 1, 2010 and September 30, 2015 at the Institute of Biomedical Research and Innovation (Kobe, Japan). All the patients were scanned using CT (Bright Speed; GE Healthcare UK Ltd, Little Chalfont, Buckinghamshire, UK). CT image thickness was $2.5 \mathrm{~mm}$. Examinations were performed based strictly on clinical indications alone. Informed consent was obtained from each patient before the procedure was performed. The biopsies were performed by experienced physicians. This retrospective 

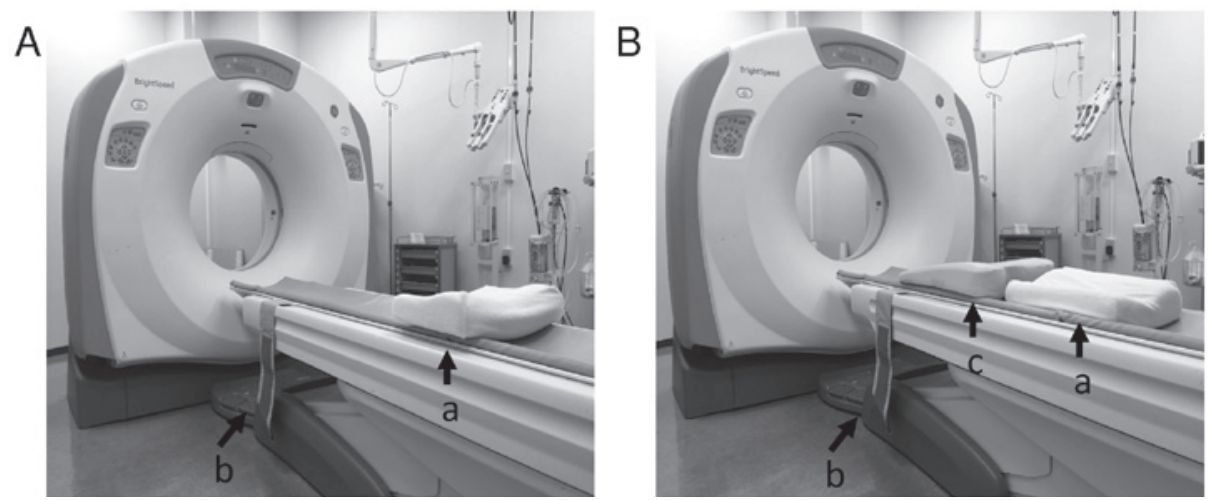

Figure 1. Patient position for CT-guided lung biopsy. CT scanner room. The operator stood at the left side of the scanner. The patient had a pulmonary lesion side. (A) Supine position headfirst, pulmonary lesion was front side of the right lung; feet first, pulmonary lesion was front side of the left lung. (B) Prone position headfirst, pulmonary lesion was back side of the left lung; feet first, pulmonary lesion was back side of the right lung; a, pillow; b, band for fixation; $\mathrm{c}$, hard cushion where the head was higher than the feet.

study was approved by the ethics committee of the Institute of Biomedical Research and Innovation (Kobe, Japan).

Procedures. Each patient underwent a percutaneous lung biopsy using conventional CT scan guidance with a co-axial image. Each patient was placed in the prone or spine position during the procedure. Before the scan was initiated, the patient practiced breath holding at deep expiration and deep inspiration.

The CT scan was used to accurately locate the lesion and determine the shortest route for the biopsy. The $\mathrm{CT}$ scan range included the typical pulmonary lesion size (i.e., 30-40 mm). The protocol specified the use of effective $110-150 \mathrm{~mA}$ at $120 \mathrm{kV}$, a 0.5 -sec rotation time, a collimation (beam width) of $12 \mathrm{~mm}$, a $14.4-\mathrm{mm}$ x-ray beam width, and a $2.5-\mathrm{mm}$ slice thickness. The lung function reconfiguration function was used and the field of view (FOV) was $22 \mathrm{~cm}$.

The biopsies were performed using a needle system with a 19-gauge introducer needle (Co-Axial Introducer Needle, Angiotech, Gainesville, FL, USA) and a 22-gauge needle for fine needle aspiration (HAKKO Sonoguide PTC, needle type-B, Hakko-Medical, Chikuma, Japan). A 20-gauge automated cutting biopsy needle with 15 - or 25-mm notches was used for the core needle biopsy (Bard Magnum, Medicon, Osaka, Japan).

The CT-guided lung biopsy examination was performed using a standard procedure. First, CT images of the lesion position in the right or left lung were obtained. The CT landmark was the center of the lesion. A radiopaque grid was placed on the patient's skin in position over the lesion, and the skin puncture point and the lesion center were determined using images that included this grid. The puncture point was determined after the operator measured the skin surface to pleura distance, the needle path distance, and the needle angle (Fig. 2A). The skin incision was made after $10 \mathrm{ml} 1 \%$ xylocaine was administered subcutaneously as a local anesthetic. The initial puncture was stopped at the pleura surface and did not puncture the pleura (Fig. 2B). CT images were obtained, and the puncture point was determined after the needle tip to lesion distance, the needle path distance, and the needle angle were measured (with punctured pleura, Fig. 2C). When skin to lesion distance was long, the operator made the puncture along the access route until the lesion was encountered. If the needle angle was incorrect, the operator changed it to an appropriate angle (with punctured lesion, Fig. 2D). Specimens obtained were immediately immersed in $10 \%$ buffered formalin solution. A chest CT scan of the lung apices to the diaphragm was routinely performed at the end of the procedure to detect the presence of pneumothorax or intrapulmonary hemorrhage.

Measurement yield. The angle between the pleura and needle, and the maximum diameters between the pleura and needle tip on the lung window were measured (window wide, 1700; window level, -600). Fig. 3 shows the results for the needle angle, which was measured from the operator's position (performed during sessions 5 or 6). Lesions abutting the pleural surface were excluded from the analysis. Patients with and without pneumothorax were separated into two groups. Level 1 pneumothorax occurred at the cranial lung apex at the clavicle level. Level 2 was between levels 1 and 3. Level 3 pneumothorax was a complete or near complete collapse of the lung.

Statistical analysis. The independent risk factors for pneumothorax were determined using multivariate logistic regression analysis. Data on multiple variables related to the patient, lesion, and procedure were collected for statistical analysis. Linear two-sided t-tests or $\chi^{2}$-tests were performed for the between-variable analyses. $\mathrm{P}<0.05$ was considered to indicate a statistically significant result.

\section{Results}

Patient characteristics, and multivariate and univariate analyses. We retrospectively analyzed the data from 325 consecutive patients who underwent CT-guided lung biopsies from April 1, 2010 to September 30, 2015. The CT dose index (CTDIw) (22) was 0.49 $\pm 0.14 \mathrm{mGy}$ (range, 0.28-1.19 mGy). Table I presents the results for patient demographic characteristics and the results of further multivariate analyses. Table II presents the results of univariate analyses used to investigate associations between risk factors and pneumothorax development. Biopsy-related pneumothorax occurred in $49.2 \%$ $(160 / 325)$ of the procedures. A total of $5.5 \%$ (18/325) of the procedures were discontinued as a result of the development 

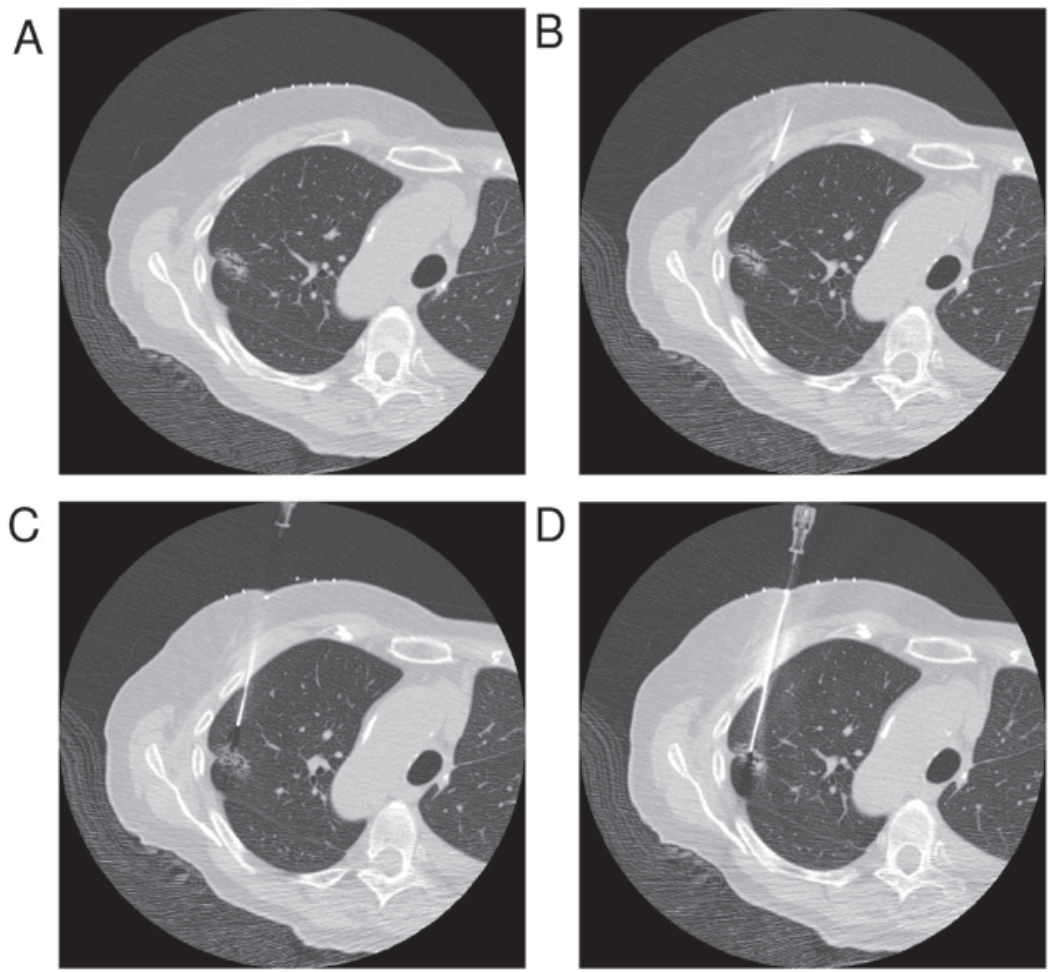

Figure 2. Conventional CT-guided biopsy method. (A) The center of the lesion was positioned at the CT landmark using a radiopaque grid placed on the patient's skin. (B) The initial puncture was stopped when the surface of the pleura was reached. Biopsies were performed to avoid ribs, bullae, vessels, and fissures. (C) If the lesion was on the extended course of the needle track, the biopsy procedure was continued. If the nodule was not on the extended course of the needle track, the course or puncture site was changed. (D) Specimens obtained were immediately immersed in $10 \%$ buffered formalin solution.

Table I. Patient and biopsy characteristics.

\begin{tabular}{lc}
\hline Patient & Values \\
\hline Age (years) & $69.3 \pm 11.1$ \\
Sex, no. & \\
Male & $180(55)$ \\
Female & $145(45)$ \\
Biopsy needle angle; degree & \\
Session 5 & $70.9 \pm 24.0$ \\
Session 6 & $71.8 \pm 22.8$ \\
From session 5 to session 6 & $5.1 \pm 8.1$ \\
Lobe, no. & \\
Upper & $133(40.9)$ \\
Middle & $48(14.7)$ \\
Lower & $144(44.3)$ \\
\end{tabular}

All values are presented as the mean \pm SD or No. $(\%)$. SD, standard deviation.

of pneumothorax. Fig. 4 presents the results of univariate analyses used to investigate the relationships between lung lobe and pneumothorax development; $40.8 \%$ occurred in the upper (58/142), $17.6 \%$ in the middle $(25 / 142)$, and $41.5 \%$ in the lower (59/142) lung lobes.

The angle of biopsy needle and pleura. Table II presents the results for the relationship between needle angle and the pleura,

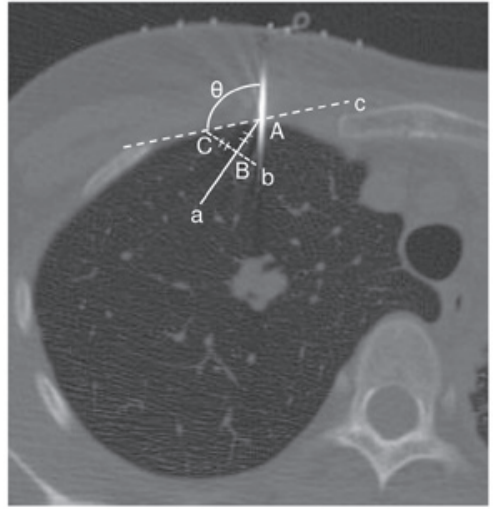

Figure 3 . The angle of the needle. The angle measured a pulmonary surface using the simple tangent line method on a CT image. Point A was the point where the needle crossed with the pulmonary surface. Point B was $10 \mathrm{~mm}$ into the pulmonary surface from point $\mathrm{A}$. Line, a, was between point $\mathrm{A}$ and point B. Line, b, was straight through line, a, through point B. Line, b, was at a right angle to line, a. Point $\mathrm{C}$ was $10 \mathrm{~mm}$ from point $\mathrm{B}$ on the pulmonary surface. Line, $\mathrm{c}$, was the line that linked point $\mathrm{C}$ to point $\mathrm{A}$. The needle angle $(\theta)$ was measured $\left(^{\circ}\right)$ between the needle and the pulmonary surface.

and pneumothorax development. Of those procedures in session $5,73.2 \pm 22.1$ had no pneumothorax and $70.5 \pm 23.9 \%$ experienced pneumothorax $(\mathrm{P}=0.5195) ; 70.2 \pm 21.4$ were discontinued because pneumothorax developed $(\mathrm{P}=0.6841)$. Of those procedures in session $6,72.6 \pm 22.7 \%$ had no pneumothorax and $71.0 \pm 22.9 \%$ had pneumothorax $(\mathrm{P}=0.5411)$, while $72.4 \pm 18.9 \%$ were discontinued because pneumothorax developed $(\mathrm{P}=0.9839)$.

When the needle angle was $<90^{\circ}, 40.3 \%(131 / 325)$ of the procedures were performed without the development 
Table II. Results of univariate analyses to determine risk factors for pneumothorax.

\begin{tabular}{|c|c|c|c|c|c|}
\hline Factors & $\begin{array}{l}\text { Without pneumothorax } \\
\qquad(\mathrm{n}=165)\end{array}$ & $\begin{array}{l}\text { With pneumothorax } \\
\qquad(\mathrm{n}=142)\end{array}$ & $\begin{array}{l}\text { Discontinuance } \\
\qquad(\mathrm{n}=18)\end{array}$ & P-value ${ }^{a}$ & P-value ${ }^{b}$ \\
\hline \multicolumn{6}{|l|}{ Patient } \\
\hline Age, year & $69.3 \pm 9.3$ & $69.7 \pm 11.3$ & $71.0 \pm 7.8$ & 0.6831 & 0.4404 \\
\hline \multicolumn{6}{|l|}{ Sex } \\
\hline Female & $73(22.4)$ & $80(28.0)$ & $11(3.4)$ & & \\
\hline Male & $92(28.3)$ & $62(21.2)$ & $7(2.1)$ & & \\
\hline \multicolumn{6}{|l|}{ Lobe } \\
\hline Upper & $72(22.5)$ & $58(17.8)$ & $4(1.2)$ & & \\
\hline Middle or Lingular & $21(6.5)$ & $25(7.7)$ & $1(0.3)$ & & \\
\hline Lower & $72(22.2)$ & $59(18.2)$ & $13(4.0)$ & & \\
\hline Upper-Middle & & & & 0.2855 & 0.8928 \\
\hline Upper-Lower & & & & 0.5339 & 0.0387 \\
\hline Middle-Lower & & & & 0.5264 & 0.1827 \\
\hline \multicolumn{6}{|l|}{ The angle of needle } \\
\hline Session 5 & $73.2 \pm 22.1$ & $70.5 \pm 23.9$ & $70.2 \pm 21.4$ & 0.5195 & 0.6841 \\
\hline Session 6 & $72.6 \pm 22.7$ & $71.0 \pm 22.9$ & $72.4 \pm 18.9$ & 0.5411 & 0.9839 \\
\hline From session 6 to 5 & $4.6 \pm 4.49$ & $6.7 \pm 10.3$ & $3.55 \pm 2.80$ & 0.1817 & 0.4584 \\
\hline
\end{tabular}

Values are presented as the mean $\pm \mathrm{SD}$ or no. $(\%)$. ${ }^{\mathrm{a}} \mathrm{P}$-value between without and with pneumothorax. ${ }^{\mathrm{b}} \mathrm{P}$-value between without pneumothorax and discontinuance.

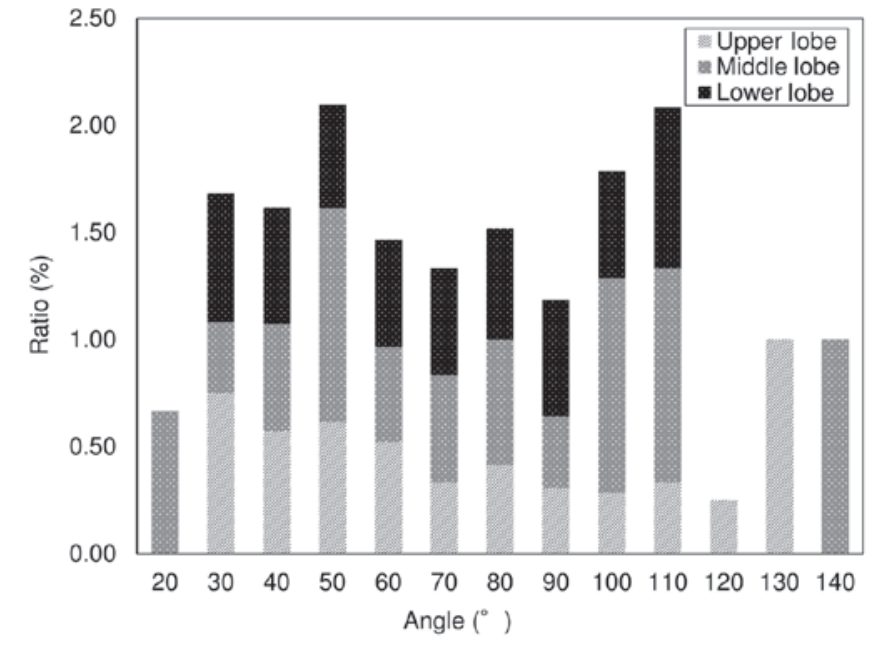

Figure 4. Ratios, location of pneumothorax at a specific needle angle.

of pneumothorax and $40.0 \%(130 / 325)$ with the development of pneumothorax. A total of $4.3 \%(14 / 325)$ of the procedures were discontinued as a result of pneumothorax development. Fig. 5 shows the results of the univariate analyses to determine the relationship between needle angle and pneumothorax development. Of those, $23.1 \%$ of the procedures were in session 5 (37/160), 27.5\% were in session 6 (44/160), 36.9\% were post CT (59/160), and $12.5 \%$ were X-P (20/160). Fig. 6 presents the results of the univariate analyses to summarize pneumothorax severity and discontinuation of the procedure; $59.4 \%$ of the patients had mild $(95 / 160), 25.0 \%$ had moderate (40/160), and $7.5 \%$ had severe (12/160) pneumothorax. A total of $11.2 \%(18 / 160)$ of the procedures were discontinued.

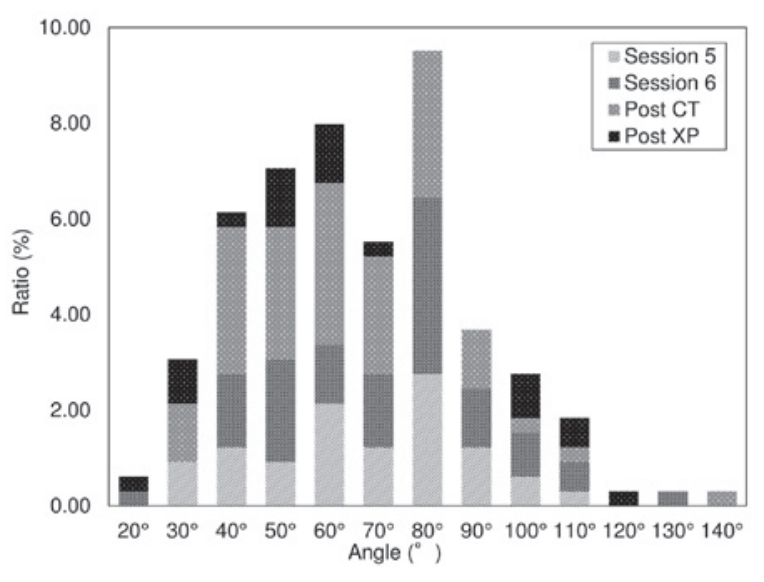

Figure 5. Ratios, stage of pneumothorax development.

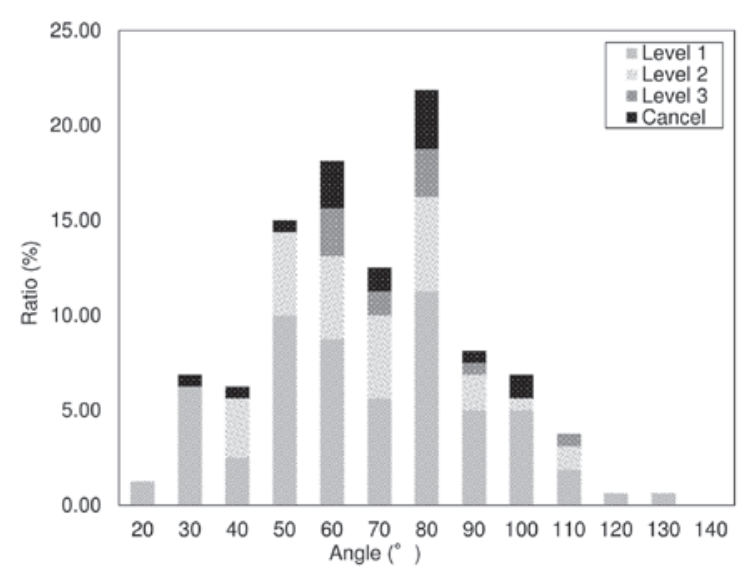

Figure 6. Ratios, pneumothorax development at a specific level in the thorax. 
Discontinuation of CT lung biopsy. The lung biopsy was discontinued in 18 cases; $2.5 \%$ (4/160) of these had upper, $0.6 \%$ $(1 / 160)$ had middle, and $8.1 \%$ (13/160) had lower lung lobe pneumothorax. Of those procedures that were discontinued because pneumothorax developed $33.3 \%(6 / 18)$ were a level 1 , $50.0 \%(9 / 18)$ were a level 2 , and $16.7 \%(3 / 18)$ were a level 3 pneumothorax. A total of $61.1 \%$ (11/18) of the procedures were in session 5 and $38.9 \%(7 / 18)$ in session 6 .

\section{Discussion}

The incidence of pneumothorax was $49.2 \%(160 / 325)$ in this study population. CT-guided lung biopsy becomes more difficult or may even be discontinued if pneumothorax develops. Two important risk factors that affect pneumothorax rate are the distance from the pleura to the lesion and the distance from the pleura to the needle tip.

Of the biopsies that were discontinued because of pneumothorax development, $2.5 \%$ was upper lung lobe, $0.6 \%$ was middle lobe, and $8.1 \%$ was lower lobe biopsies. The lower lung lobe is, in particular, affected by diaphragmatic movement. During the procedure, the patient was instructed to hold their breath to suppress diaphragmatic movement. However, it is impossible to completely stop diaphragmatic movement; breath holding will only reduce it. Increased motion of the lower lobe during respiration may cause inaccurate placement of the biopsy needle into the lesion. Janssens et al reported that females aged $>65$ years and males aged $>75$ years have age-related reduced respiratory muscle strength and experience difficulty maintaining a held breath for extended periods of time (21). We hypothesize that the patients affected by pneumothorax in this study had a reduced ability to maintain breath holding for the required length of time. The patients were in a moving gantry during sessions 3,5 , and 6 , and may not have heard the operator's instructions to hold their breath during inspiration or expiration. Hanley et al (23) reported that when diaphragmatic movement decreases, it suppresses the abdomen, and the patient may have difficulty stopping their breath. Wong et al (24) reported that diaphragmatic movement decreases are greater if mouth breathing, not nose breathing, is used. The incidence of pneumothorax was likely lower in our patient population compared with other patient populations because we instructed patients to use this technique during the procedure.

As the patient was in an unnatural position during CT, the patient was instructed to practice breath holding at the end of deep inspiration and expiration. We also used CT images to confirm that the access route bypassed the ribs and arteries. The angle between the biopsy needle and pleura was $<90^{\circ}$ in $84.6 \%(275 / 325)$ of the procedures. The positioning (supine, prone, or oblique) was maintained to increase the ease with which the operator could insert the needle. The angle between the needle and pleura was $<90^{\circ}$ in the anterior-posterior and right-left positions. During the biopsy, the operator stood to the left side of the CT couch. We tried to acquire the target angle by setting a pillow or a towel between the patient and the couch (Fig. 1). The angle was typically between $40^{\circ}$ and $90^{\circ}(76.6 \%, 249 / 325)$ because the operator used this method to secure a puncture access route (e.g., towels were placed under the clavicle when the lesion was in the lung apex).
More accurate examination of the access route from the skin surface to the lesion may result from careful examination of the 3-D image of the relationships between the lung, lesion, and bone before the day of the procedure. To reduce the incidence of pneumothorax, the positioning of the patient, the acquiring route, and the level of experience of the operator should be reviewed (17). A hold system (Vac-Lok Bean Bag: MEDTEC, Orange City, IA, USA) is an ideal system for patient positioning (25). It has high repeatability and is relatively comfortable for the patient. The patient is also likely to be more cooperative during the biopsy if breath holding is practiced starting from the day before the procedure.

Our study was limited by the use of retrospective design, because unexamined risk factors may have been present that were associated with discontinuing the procedure as a result of pneumothorax development. Our data also did not include information on tumor-positive rates. However, these results contribute to increased understanding regarding the conditions that should be present to increase the probability of successful CT-guided lung biopsy.

In conclusion, this study revealed the factors that affect the risk of developing pneumothorax and that affect the risk that pneumothorax may result in discontinuation of CT-guided lung biopsy (e.g., needle angle, lobe where the lesion is present, and patient positioning). The success rate of CT-guided lung biopsy can be improved if the factors that affect pneumothorax development are considered. The access route is simple to locate, easy to puncture by the operator, and diaphragmatic movement can be successfully managed.

\section{References}

1. Diederich S, Wormanns D and Heindel W: Low-dose CT: New tool for screening lung cancer? Eur Radiol 11: 1916-1924, 2001.

2. Henschke CI, Yankelevits DF, Libby DM, McCauley D, Pasmantier M, Altorki NK, Smith JP and Miettinen OS: Early lung cancer action project: Annual screening using single helical CT. Ann N Y Acad Sci 952: 124-134, 2001.

3. Henschke CI, Yankelevits DF, Mirtcheva R, McGuinness G, McCauley D and Miettinen OS; ELCAP Group: CT screening for lung cancer: Frequency and significance of part-solid and nonsolid nodules. AJR Am J Roentgenol 178: 1053-1057, 2002.

4. Henschke CI, Yankelevits DF, Libby DM and Kimmel M: CT screening for lung cancer: The first ten years. Cancer J 8 (Suppl 1): S47-S54, 2002.

5. Pastorino U, Bellomi M, Landoni C, De Fiori E, Arnaldi P, Picchio M, Pelosi G, Boyle P and Fazio F: Early lung cancer detection with spiral CT and positron emission tomography in heavy smokers: 2-year results. Lancet 362: 593-597, 2003.

6. Bastarrika G, García-Velloso MJ, Lozano MD, Montes U, Torre W, Spiteri N, Campo A, Seijo L, Alcaide AB, Pueyo J, et al: Early lung cancer detection using spiral computed tomography and positron emission tomography. Am J Respir Crit Care Med 171: 1378-1383, 2005.

7. Harter LP, Moss AA, Goldberg HI and Gross BH: CT-guided fine-needle aspirations for diagnosis of benign and malignant disease. AJR Am J Roentgenol 140: 363-367, 1983.

8. Li H, Boiselle PM, Shepard JO, Trotman-Dickenson B and McLoud TC: Diagnostic accuracy and safety of CT-guided percutaneous needle aspiration biopsy of the lung: Comparison of small and large pulmonary nodules. AJR Am J Roentgenol 167: 105-109, 1996

9. Klein JS and Zarka MA: Transthoracic needle biopsy: An overview. J Thorac Imaging 12: 232-249, 1997.

10. Takeshita J, Masago K, Kato R, Hata A, Kaji R, Fujita S and Katakami N: CT-guided fine needle aspiration and core needle biopsy of pulmonary lesions: A single-center experience on 750 biopsies in Japan. AJR Am J Roentgenol 204: 29-34, 2015. 
11. Tomiyama N, Yasuhara Y, Nakajima Y, Adachi S, Arai Y, Kusumoto M, Eguchi K, Kuriyama K, Sakai F, Noguchi M, et al: CT-guided needle biopsy of lung lesions: A survey of severe complication based on 9783 biopsies in Japan. Eur J Radiol 59: 60-64, 2006.

12. Poulou LS, Tsagouli P, Ziakas PD, Politi D, Trigidou R and Thanos L: Computed tomography-guided needle aspiration and biopsy of pulmonary lesions: A single-center experience in 1000 patients. Acta Radiol 54: 640-645, 2013.

13. Hiraki T, Mimura H, Gobara H, Iguchi T, Fujiwara H, Sakurai J, Matsui Y, Inoue D, Toyooka S, Sano Y and Kanazawa S: CT fluoroscopy-guided biopsy of 1,000 pulmonary lesions performed with 20-gauge coaxial cutting needles: Diagnostic yield and risk factors for diagnostic failure. Chest 136: 1612-1617, 2009.

14. Tsukada H, Satou T, Iwashima A and Souma T: Diagnostic accuracy of CT-guided automated needle biopsy of lung nodules. AJR Am J Roentgenol 175: 239-243, 2000.

15. Laurent F, Latrabe V, Vergier B, Montaudon M, Vernejoux JM and Dubrez J: CT-guided transthoracic needle biopsy of pulmonary nodules smaller than $20 \mathrm{~mm}$ : Results with an automated 20 -gauge coaxial cutting needle. Clin Radiol 55: 281-287, 2000.

16. Saji H, Nakamura H, Tsuchida T, Tsuboi M, Kawate N, Konaka C and Kato $\mathrm{H}$ : The incidence and the risk of pneumothorax and chest tube placement after percutaneous CT-guided lung biopsy: The angle of the needle trajectory is a novel predictor. Chest 121 : 1521-1566, 2002.

17. Yeow KM, Su IH, Pan KT, Tsay PK, Lui KW, Cheung YC and Chou AS: Risk factors of pneumothorax and bleeding: Multivariate analysis of 660 CT-guided coaxial cutting needle lung biopsies. Chest 126: 748-754, 2004.

18. Kazerooni EA, Lim FT, Mikhail A and Martinez FJ: Risk of pneumothorax in CT-guided transthoracic needle aspiration biopsy of the lung. Radiology 198: 371-375, 1996.
19. Topal U and Ediz B: Transthoracic needle biopsy: Factors effecting risk of pneumothorax. Eur J Radiol 48: 263-267, 2003.

20. Yeow KM, Tsay PK, Cheung YC, Lui KW, Pan KT and Chou AS: Factors affecting diagnostic accuracy of CT-guided coaxial cutting needle lung biopsy: Retrospective analysis of 631 procedures. J Vasc Interv Radiol 14: 581-588, 2003.

21. Janssens JP, Pache JC and Nicod LP: Physiological changes in respiratory function associated with ageing. Eur Respir J 13: 197-205, 1999.

22. Zompatori M, Fasano L, Mazzoli M, Sciascia N, Cavina M, Pacilli AM and Paioli D: Spiral CT evaluation of pulmonary emphysema using a low-dose technique. Radiol Med 104: 13-24, 2002 (In English, Italian).

23. Hanley J, Debois MM, Mah D, Mageras GS, Raben A, Rosenzweig K, Mychalczak B, Schwartz LH, Gloeggler PJ, Lutz W, et al: Deep inspiration breath-hold technique for lung tumors: The potential value of target immobilization and reduced lung density in dose escalation. Int J Radiat Oncol Biol Phys 45: 603-611, 1999

24. Wong JW, Sharpe MB, Jaffray DA, Kini VR, Robertson JM, Stromberg JS and Martinez AA: The use of active breathing control $(\mathrm{ABC})$ to reduce margin for breathing motion. Int $\mathrm{J}$ Radiat Oncol Biol Phys 44: 911-919, 1999.

25. Takayama K, Mizowaki T, Kokubo M, Kawada N, Nakayama H, Narita Y, Nagano K, Kamino Y and Hiraoka M: Initial validations for pursuing irradiation using a gimbals tracking system. Radiother Oncol 93: 45-49, 2009. 